In Crescendo. Institucional. 2015; 6(2): 11-21

Fecha de recepción: 24 de noviembre de 2015

Fecha de aceptación: 1 de diciembre de 2015

\title{
TRANSMISIÓN TRANSGENERACIONAL DE LA VIOLENCIA DE PAREJA Y FUNCIONALIDAD FAMILIAR DE HOMBRES Y MUJERES DE LA CIUDAD DE TRUjILLO
}

\section{TRANSGENERATIONAL TRANSMISSION OF INTIMATE PARTNER VIOLENCE AND FAMILY FUNCTIONALITY OF MEN AND WOMEN IN THE CITY OF TRUjILlO} Paúl Alan Arkin Alvarado García*

\begin{abstract}
RESUMEN
T a violencia de pareja es considerada actualmente como un problema de salud pública, Lfenómeno extendido en el espacio y el tiempo. Por tanto, el objetivo del presente trabajo fue determinar la relación entre la transmisión transgeneracional de la violencia de pareja y la funcionalidad familiar, para lo cual se realizó un estudio exploratorio de diseño no experimental, descriptivo correlacional en 384 personas, hombres y mujeres, a quienes se les administró la entrevista semiestructurada de Salazar para evaluar la transmisión transgeneracional de la violencia de pareja, y el cuestionario de percepción de la funcionalidad familiar APGAR para evaluar la funcionalidad familiar. Se determinó la relación entre la transmisión transgeneracional de la violencia de pareja y la funcionalidad familiar, con resultados estadísticamente significativos $(\mathrm{p}<.05)$. Los entrevistados con familias disfuncionales presentaron mayor violencia de pareja en las tres generaciones evaluadas. La disfuncionalidad familiar y la violencia de pareja en las generaciones pasadas son un factor para la ocurrencia de violencia de pareja.
\end{abstract}

Palabras clave: Transmisión transgeneracional, violencia de pareja, funcionamiento familiar.

* Licenciado en Psicología. Escuela de Psicología. Universidad Señor de Sipán. Email: agarciap@crece. uss.edu.pe 


\begin{abstract}
Nowadays, intimate partner violence, is as a public health problem, a widespread phenomenon not only in space but also in time, which is why the aim of this work was determining the relationship between the intergenerational transmission of intimate partner violence and family functioning. It was made an exploratory study of nonexperimental, descriptive correlational design in 384 people, men and women, which were administered Salazar's semistructured interview to assess the transgenerational transmission of intimate partner violence, and family functioning APGAR questionnaire was conducted to evaluate the functionality of the family.

It was determined the relation between the transgenerational transmission of intimate partner violence and family functioning with a significance level of $p<0,05$. Respondents with dysfunctional families had higher intimate partner violence in the three studied generations. The family dysfunction and intimate partner violence in past generations are risk factors for intimate partner violence.
\end{abstract}

Keywords: Transgenerational transmission of intimate partner violence, family functioning.

\title{
INTRODUCCIÓN
}

En la actualidad, la Organización Mundial de la Salud considera la violencia de pareja como un problema de salud pública de influencia global. Las repercusiones de este fenómeno superan el microcontexto individual y alcanzan el macrocontexto social, lo que repercute no solo en la salud de un individuo y su familia, sino también en la salud mental de la sociedad. Así, un estudio multipaís encontró que de un $15 \%$ a $71 \%$ de mujeres de entre 15 a 49 años de edad, reportaron haber experimentado violencia física o sexual en algún momento de sus vidas (OMS, 2005).

En el Perú, según la encuesta ENDES, un millón de mujeres fueron agredidas por su esposo o compañero; 87000 por mes; 2853 por día; 119 por hora y 2 por minuto (ENDES, 2004). En la región La Libertad, durante el año 2012 hubo un incremento de este fenómeno como lo muestran las cifras del Ministerio de la Mujer y Poblaciones Vulnerables, especificando que esta región lidera junto con Lima, el mayor número de denuncias por agresiones físicas contra mujeres (La Libertad, 2012).

Diversas investigaciones determinan que la exposición a violencia de pareja genera la posibilidad de recrearla en el futuro, como Mukherjee y Parasuraman, quienes entrevistaron 28904 parejas, y encontraron que aquellas personas que fueron testigos de la violencia entre los padres durante su infancia perpetuaban actos de violencia contra sus 
parejas en la edad adulta (Mukherjee y Parasuraman, 2012). Cannon et al. (2009) determinaron en una muestra de 1288 mujeres que las hijas de mujeres maltratadas tienen mayor probabilidad de vivenciar episodios de violencia de pareja. En otra investigación, con pacientes con intento de suicido hospitalizados por violencia de pareja, encontraron que las mujeres con disfuncionalidad familiar declararon mayor maltrato psicológico por parte de sus parejas (Heru, Stuart y Recupero, 2007). Por su parte Bogat, Levendosky y Davidson (2002) evaluaron los efectos de la violencia durante el embarazo y el primer año de vida, concluyendo que las hijas de las madres que experimentaron violencia durante el embarazo, vivenciaron episodios de violencia en sus relaciones de pareja.

Sin embargo, la mayoría de las investigaciones se enfocan en estudios de dos generaciones, sin tener evidencia de lo que sucede cuando la distancia generacional es más larga. Al hablar de tres generaciones o más, entra a tallar un concepto nuevo que se denomina transmisión transgeneracional, que suele definirse, según Tisseron, Torok y Rand (1997), como la influencia de los contenidos psíquicos a través de la sucesión de las generaciones. En otras palabras, el contenido psíquico de los hijos queda marcado por el contenido de los padres, abuelos o por otro antepasado familiar que tuvo gran peso identificatorio. Estos patrones se van repitiendo a través de las generaciones en las familias, y son asimiladas por los miembros, que continúan su reproducción, en relación al funcionamiento de las relaciones y la estructura familiar como soluciones intentadas y recreadas a través de las relaciones, jerarquías, roles y mitos familiares, donde la identificación de estos patrones de violencia puede evitar seguir repitiendo estas pautas en el presente y transmitirlas en el futuro (Urzagasti, 2006).

Bajo este contexto, se realizó la presente investigación con el objeto de determinar la relación entre la transmisión transgeneracional de la violencia de pareja y la funcionalidad familiar de hombres y mujeres de la ciudad de Trujillo.

\section{MATERIALES Y MÉTODOS}

\section{Muestra}

La muestra de 384 personas se distribuyó proporcionalmente mediante un muestreo aleatorio estratificado en 184 hombres y 200 mujeres.

\section{Instrumentos}

Para determinar la transmisión transgeneracional de la violencia de pareja se utilizó la entrevista semiestructurada de Salazar (2010), la cual indaga información sobre la presencia o ausencia de la violencia de pareja en la generación actual, así como los antecedentes del fenómeno en la generación de los padres y de los abuelos. Por otro lado, para evaluar el funcionamiento familiar, se utilizó el cuestionario de percepción del funcionamiento familiar APGAR, el cual evalúa cómo el individuo percibe la funcionalidad de su familia, a través de cinco dimensiones de adaptabilidad, asociación, desarrollo, afecto y resolución, correspondientes a cada uno de sus ítems, los cuales se puntúan a través de una escala de 
Likert con tres categorías (nunca, a veces siempre, correspondientes a 2, 1 y 0 puntos), pudiéndose obtener resultados de funcionalidad familiar ( 7 a 10 puntos) y disfuncionalidad familiar (0 a 6 puntos). Para la presente investigación se realizó un estudio piloto en 36 sujetos para verificar la validez y confiabilidad de cada uno de los instrumentos en la población local, para lo cual se usó el método ítem-test (Borrego y Vásquez, 2008), donde puntajes mayores o iguales a 0,21 son considerados como válidos. También se realizó el cálculo de confiabilidad por el método de las mitades, donde coeficientes mayores o iguales a 0,80 indican la confiabilidad de la prueba. Los resultados de validez y confiabilidad de ambos instrumentos se observan en las tablas 1 y 2 respectivamente.

Tabla 1. Consolidado de reactivos según la validez Ítem-Test de instrumentos de evaluación

\begin{tabular}{ccccc} 
INSTRUMENTO & $\begin{array}{c}\text { ENTREVISTA SEMIESTRUCTURADA } \\
\text { DE SALAZAR }\end{array}$ & \multicolumn{2}{c}{ CUESTIONARIO APGAR } \\
ÍTEM & Coeficiente validez & Resultado & Coeficiente validez & Resultado \\
\hline 1 & 0,972093312 & VÁLIDO & 0,314276393 & VÁLIDO \\
2 & 0,960372989 & VÁLIDO & 0,29889899 & VÁLIDO \\
3 & 0,972093312 & VÁLIDO & 0,281373059 & VÁLIDO \\
4 & 0,960372989 & VÁLIDO & 0,398581641 & VÁLIDO \\
5 & 0,972093312 & VÁLIDO & 0,388944578 & VÁLIDO \\
6 & 0,960372989 & VÁLIDO & & \\
7 & 0,972093312 & VÁLIDO & & \\
8 & 0,960372989 & VÁLIDO & & \\
9 & 0,972093312 & VÁLIDO & & \\
10 & 0,960372989 & VÁLIDO & & \\
11 & 0,655381093 & VÁLIDO & & \\
12 & 0,960372989 & VÁLIDO & & \\
13 & 0,972093312 & VÁLIDO & & \\
\hline
\end{tabular}

Tabla 2. Confiabilidad de Instrumentos de evaluación

\begin{tabular}{cccc}
\hline INSTRUMENTO & $\begin{array}{c}\text { COEFICIENTE DE } \\
\text { PEARSON }\end{array}$ & $\begin{array}{c}\text { COEFICIENTE DE } \\
\text { SPEARMAN BROWN }\end{array}$ & RESULTADO \\
\hline $\begin{array}{c}\text { Entrevista Semiestruc- } \\
\text { turada de Salazar }\end{array}$ & 0,8589 & 0,9240 & CONFIABLE \\
Cuestionario APGAR & 0,8298 & 0,9069 & CONFIABLE \\
\hline
\end{tabular}

\section{Diseño y procedimiento}

Se realizó un estudio exploratorio de diseño no experimental, descriptivo correlacional, para lo cual se entrevistó al paso a hombres y mujeres de los diferentes distritos de la ciudad de Trujillo. Las entrevistas se realizaron en los principales puntos de conglomeración popular (plazas de armas, principales calles y centros comerciales). Participaron en el estudio aquellas personas que accedieron voluntariamente y que hayan tenido o tengan (en el momento del estudio) una relación de pareja. A los participantes, se les explicó el 
objetivo e importancia de la investigación. Luego de su aceptación, firmaron el consentimiento informado para hacer uso de los datos obtenidos, manteniendo y garantizando la confidencialidad y anonimato. A continuación se les repartió la entrevista semiestructurada de Salazar, y el cuestionario APGAR, explicándoles cuidadosamente las instrucciones. Al final se recogieron los instrumentos, y se les agradeció por su participación voluntaria. Finalmente, los resultados fueron analizados mediante el software estadístico SPSS versión 20.

\section{Análisis estadístico}

A fin de conocer las principales características de las puntuaciones, se calcularon las frecuencias expresadas en porcentajes; adicionalmente se aplicó la prueba de bondad de ajuste a la curva normal de Kolmogorov-Smirnov a fin de poder seleccionar los estadísticos a utilizar, y se decidió por la prueba no paramétrica de Chi cuadrado $\left(\mathrm{X}^{2}\right)$ para determinar la relación entre las variables y la prueba de Odds ratio para determinar el factor de riesgo. Para procesar los datos se utilizaron los programas MS Excel 2010, SPSS 20.0.

\section{RESULTADOS}

Gráfico 1. Transmisión Transgeneracional de la Violencia de Pareja y Funcionalidad Familiar de hombres y mujeres de la ciudad de Trujillo. $\mathrm{p}<0.05$ (prueba de $\mathrm{X}^{2}$ )

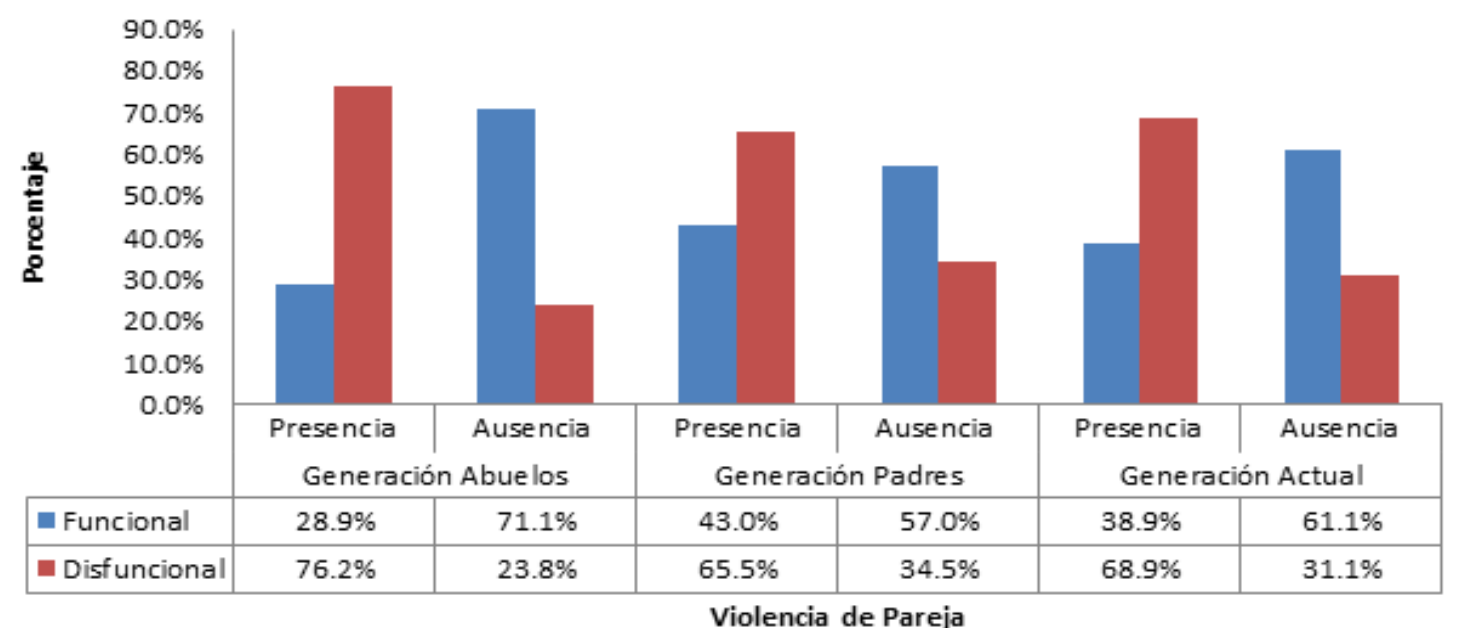

Tabla 1. Niveles de violencia de pareja en las tres generaciones y funcionalidad familiar según el sexo de los entrevistados.

\begin{tabular}{cccccccc}
\multirow{2}{*}{ Sexo } & Funcionalidad & \multicolumn{5}{c}{ Violencia de pareja } \\
\cline { 3 - 7 } & familiar & & \multicolumn{2}{c}{ Generación de los abuelos } & Generación de los padres & Generación actual \\
\cline { 3 - 7 } & & Presencia & Ausencia & Presencia & Ausencia & Presencia & Ausencia \\
\multirow{2}{*}{ Hombres } & Funcional & $26,7 \%$ & $73,3 \%$ & $17,8 \%$ & $82,2 \%$ & $20,0 \%$ & $80,0 \%$ \\
& Disfuncional & $80,6 \%$ & $19,4 \%$ & $56,1 \%$ & $43,9 \%$ & $54,7 \%$ & $45,3 \%$ \\
\multirow{2}{*}{ Mujeres } & Funcional & $29,8 \%$ & $70,2 \%$ & $53,8 \%$ & $46,2 \%$ & $47,1 \%$ & $52,9 \%$ \\
& Disfuncional & $69,8 \%$ & $30,2 \%$ & $79,2 \%$ & $20,8 \%$ & $89,6 \%$ & $10,4 \%$ \\
\hline
\end{tabular}

$\mathrm{p}<0,05$ (prueba de $\mathrm{X}^{2}$ ) 
Tabla 2. Factores de riesgo para la ocurrencia de violencia de pareja en la generación actual

\begin{tabular}{ccc} 
VARIABLES & (OR) & IC (95 \%) \\
\hline Disfuncionalidad familiar & 7,880 & $4,953-12,536$ \\
Violencia en generación de los abuelos & 2,525 & $2,657-3,848$ \\
Violencia en generación de los padres & 3,482 & $2,265-5,352$ \\
\hline
\end{tabular}

\section{DISCUSIÓN}

La violencia de pareja es un fenómeno de talla mundial que repercute no solo en los distintos estratos sociales y espacios geográficos, sino también sobrepasa las barreras generacionales. Tiene su génesis en la unidad básica de la sociedad, la familia, espacio donde se construyen también los límites y las dinámicas multigeneracionales (Fuentealba, 2007). Así, la familia al ser un agente de la reproducción social es también uno de los principales generadores de conductas interpersonales a nivel social entre sus miembros, y la calidad de aprendizaje que se dé en este ámbito va a repercutir en las futuras generaciones familiares, ya sea de manera positiva o negativa (Urzagasti, 2006). En este sentido, como se observa en el gráfico 1 , los individuos con disfuncionalidad familiar presentan mayores niveles violencia de pareja en la generación de los abuelos con 76,2 \%, generación de los padres con 65,5 \%, y generación actual con 68,9 \%, lo que muestra una constante de más 60 \% en la manifestación de la violencia de pareja en el tiempo. Esto evidencia una transmisión de la violencia de pareja, mientras que los individuos con funcionalidad familiar presentaron niveles más altos de no violencia de pareja en las tres generaciones evaluadas, sea generación de abuelos $71,1 \%$, generación de los padres $57 \%$ y generación actual $61,1 \%$. Todas estas medidas muestran significancia estadística con $\mathrm{p}<0,05$ en la prueba estadística de Chi cuadrado, que comprueba la hipótesis de que las variables transmisión transgeneracional de la violencia de pareja y funcionalidad familiar están relacionadas significativamente.

Si bien se denota una relación entre violencia de pareja a través de las generaciones y funcionalidad familiar, esto también pueden verse influenciados según la funcionalidad por género, como se observa en la tabla 1 , donde las mujeres con familias disfuncionales expresan niveles de violencia de pareja de 69,8 \% en la generación de los abuelos, 79,2 \% en generación de los padres y 89,6 \% en la generación actual; mientras los hombres con familia disfuncional alcanzan niveles de violencia de pareja de 80,6 \%, 56,1 \% y $54,7 \%$ en las generaciones respectivas. Los hombres con familia disfuncional alcanzaron los niveles más altos de ausencia de violencia de pareja en las generaciones evaluadas con $73,3 \%, 82,2 \%$ y 80,0 \%, respectivamente, mientras que 70,2 \% de las mujeres con familia disfuncional expresaron ausencia de violencia en la generación de sus abuelos, 46,2 \% en la generación de los padres y 52,9 \% expresaron episodios de violencia de pareja en la generación actual (relación actual o última relación). Estas medidas alcanzaron una significancia estadística de $\mathrm{p}<0,05$ en la prueba estadística de Chi cuadrado, lo que 
evidencia una relación significativa entre las variables. Cabe mencionar que la relación entre abuelos y nietos es muy diferente a la relación entre padres e hijos. Con los nietos, los abuelos suelen ser más permisivos, debido a la distancia generacional que les permite crear lazos más libres que favorece a la comunicación entre ellos (Martínez, 2010). Sin embargo, ante una situación de violencia, las mujeres se ven más dependientes de las figuras de apego (Ramírez y Ospina, 1998), como los abuelos, a quienes pueden iconizar, generando formaciones reactivas frente situaciones de violencia y por tanto permisividad en cuanto al relato de si estos fueron violentos, lo que podría explicar los altos porcentajes de ausencia de violencia que estas describen en la generación de sus ancestros. Por otro lado, los hombres frente a situaciones de violencia podrían mostrarse más inhibidos, generando conductas de violencia y resentimiento (Ramírez y Ospina, 1998) que pueden ser manifestadas desterrando las imágenes icónicas de los abuelos, lo que explicaría por qué los hombres expresan notoriamente que hubo violencia de pareja entre sus abuelos. En relación a la generación de los padres, la distancia generacional es más corta, por lo que se generan relaciones más rígidas entre estos (Martínez, 2010), lo que explicaría que hombres o mujeres con familia disfuncional expresen niveles altos de violencia de pareja. Por otro lado, las sociedades latinas tienen patrones culturales que legitiman el control del hombre sobre la mujer, donde los hombres suelen ser los victimarios y las mujeres víctimas (Expósito, 2011). Esto podría generar una identificación de las hijas en cuanto a sus madres, sintiéndose solidarias al expresar niveles más altos de violencia en la generación de sus predecesores. Respecto a la relación actual, probablemente la conducta estereotipada de supremacía del hombre haya generado una estructura social que apoya la desigualdad de los géneros que contribuye a que las mujeres soporten la mayor carga sustancial de violencia expresada en los niveles más altos de violencia de pareja. Existe además una normalización de las conductas del hombre: debido a un factor cultural, las mujeres pueden aceptar patrones de conducta abusivos sin ser conscientes completamente de ello (Expósito, 2011).

Como se observa en los hallazgos, existe una relación entre la violencia de pareja a través de las generaciones y disfuncionalidad familiar, llegando a ser esta última variable predictor de la primera. Como se observa en la tabla 2, los individuos con disfuncionalidad familiar tienen 7.880 más probabilidad de presentar violencia de pareja en la generación actual que aquellos individuos con funcionalidad familiar. Asimismo los entrevistados con presencia de violencia en la generación de los abuelos tienen 2.525 más probabilidad de presentar violencia de pareja en la generación actual que aquellas personas con ausencia de violencia en esta generación. Además, aquellos entrevistados con presencia de violencia de pareja en la generación de los padres tienen 3.482 veces más riesgo de presentar violencia de pareja en la generación actual. Todos los intervalos de confianza son mayores que 1, lo que evidencia resultados estadísticamente significativos. Algunos autores afirman que el riesgo de violencia marital está relacionado directamente con la disfunción familiar, lo que es también corroborado por Amato y DeBoer (s./a.). Asimismo diversos autores señalan que el presenciar violencia de pareja en la generación de los padres puede ser un factor de riesgo para la ocurrencia de violencia de pareja en la generación de los 
hijos (Mukherjee y Parasuraman, 2012; Cannon et al., 2009; Stith et al., 2004; Ehrensaft et al., 2003; Kwong et al., 2003).

Estas conductas violentas pueden explicarse mediante la teoría del aprendizaje social, según la cual la conducta violenta es aprendida, y probablemente se trasmite de generación en generación a través de los canales habituales como la familia, el juego, el deporte, las instituciones educativas y los medios de comunicación (Urzagasti, 2006; Straus, Gelles y Steinmetz,1980), lo que puede sucesos acaecidos en distancias generacionales cortas como los padres e hijos, o aquellas situaciones presenciales o correlatos trasmitidos de generación en generación, pero que sucede cuando las distancias generacionales son más largas o los hechos violentos no fueron presenciados o referidos, e incluso ni se tiene noción o conocimiento (secreto familiar). Ante esto, ya Freud postulaba la existencia de sentimientos que se trasmitían de generación en generación, ya sea en forma de deseos, inhibiciones o tabúes. Incluso va más allá postulando la existencia de un alma colectiva donde se encuentran recuerdos de los cuales no se tiene conciencia (Schützenberger, 2007). Así otros psicoanalistas afirmaban que ciertos actos, signos, conductas y síntomas pueden trasmitirse poseyendo literalmente a un sujeto, a modo de fantasma, alienándolo y actuando el individuo su ancestro así no lo haya conocido o sabido cómo este se haya comportado (Segoviano, 2008). A este fenómeno, también se le conoce como identificación, la cual es un proceso psicológico mediante el cual un sujeto asimila un aspecto, una propiedad, un atributo de otro y se transforma, total o parcialmente en él (Laplanche, 1996). Estas identificaciones se presentan generalmente en las generaciones jóvenes (Tapia y Pérez Vélez, 2011), lo que implicaría una repetición de situaciones o vivencias, denominadas lealtades familiares, donde las motivaciones de cada miembro de la familia están enraizadas dentro del contexto de su propia historia y de su grupo de pertenencia (Pardo, 2008), cuya naturalización o normalización en el caso de la violencia permite su transmisión, debido a que se internalizan las relaciones de dominio y forman parte del bagaje identificatorio del clan familiar y se convierte en un modo de vinculación, mediante la corporización de todos aquellos secretos que dejan huella y una señal de memoria en la persona o en su historia familiar (Aguiar, 1996). Sin duda alguna, hechos tan desestructurantes para el psiquismo, como la violencia, provocan agujeros representacionales que obstaculizan la elaboración psíquica de estas situaciones y propician su transmisión transgeneracional (Sánchez y Manzo, 2014); sin embargo, la identificación de estos patrones de violencia puede ayudar a evitar que estas pautas de conducta se sigan perpetuando en el presente y se trasmitan a las futuras generaciones (Urzagasti, 2006).

\section{CONCLUSIONES}

- Los individuos con disfuncionalidad familiar presentaron mayores niveles violencia de pareja en todas las generaciones evaluadas, en comparación con individuos con funcionalidad familiar, medidas con una significancia estadística de $\mathrm{p}<0,05$, lo que 
evidencia que las variables transmisión transgeneracional y violencia de pareja están relacionadas significativamente.

- Los niveles más altos de violencia de pareja en la generación de los abuelos lo denotan los hombres con disfuncionalidad familiar, mientras que las mujeres con disfuncionalidad familiar denotan niveles más altos de violencia de pareja en la generación de los padres y en la generación actual, medidas con una significancia estadística de $\mathrm{p}<0,05$, que evidencia que la violencia de pareja y la funcionalidad familiar según el sexo están relacionadas.

- La disfuncionalidad familiar y la presencia de violencia de pareja en las generaciones de los abuelos y padres son un factor de riesgo para la ocurrencia de violencia de pareja en la generación actual, medidas con significancia estadística.

\section{REFERENCIAS BIBLIOGRÁFICAS}

Aguiar, E. (1996). Violencia y pareja. Violencia social y derechos humanos. Buenos Aires: Instituto de Investigaciones Gino Germani, Facultad de Ciencias Sociales de la Universidad de Buenos Aires.

Amato, P., DeBoer, D. (s./a.). The Transmission of Marital Instability Across Generations: Relationship Skills or Commitment to Marriage? Journal of Marriage and Family, 63(4), 1038-1051.

Bogat, A., Levendosky, A., Davidson, W. (2002). Understanding the Intergenerational Transmission of Violence From from Pregnancy Through the First Year of Life, Final Project Report. Recuperado de: https://www.ncjrs.gov/pdffiles1/nij/grants/196681.pdf

Borrego, C., Vásquez, L. (2008). Módulo de aprendizaje de construcción de pruebas. Trujillo. Universidad César Vallejo.

Cannon, E., Bonomi, A., Anderson, M. y Rivara, F. (2009). The Intergenerational Transmission of Witnessing Intimate Partner Violence. Arch Pediatr Adolesc Med. 163 (8), 706-708.

Ehrensaft, M., Cohen, P., Brown, J., Smailes, E., Chen, H., y Johnson, J. (2003). Intergenerational Transmission of Partner Violence: A 20-Year Prospective Study. J Consult Clin Psychol., 71(4),741-753.

ENDES (2004). Encuesta demográfica y de salud familiar. Recuperado de: http://desa.inei. gob.pe/endes/recursos/endes2004-05_informe.pdf

Expósito F. (2011). Violencia de género. Mente y Cerebro, 48, 20-25.

Fuentealba, H. (2007). Funcionamiento familiar: su relación con la percepción de seguridad de los adolescentes y el desarrollo de conductas de riesgo de la salud. Recuperado de: http:// cybertesis.uach.cl/tesis/uach/2007/fmf954f/doc/fmf954f.pdf 
Heru, A., Stuart, M., y Recupero, P. (2007). Family functioning in suicidal inpatients with intimate partner violence. Prim Care Companion J Clin Psychiatry, 9(6), 413-418.

Kwong, M., Bartholomew, K., Henderson, A. y Trinke, S. (2003). The Intergenerational Transmission of Relationship Violence. Journ Fam Psych, 17(3), 288-301.

La Libertad lidera índice de violencia contra la mujer. (2012). La Industria. Recuperado de: http://laindustria.pe/trujillo/local/la-libertad-lidera-indice-de-violencia-contra-la-mujer

Laplanche, J. (1996). Diccionario de psicoanálisis. España: Paidós.

Martínez, A. (2010). Aproximación a los conflictos generados entre los abuelos cuidadores de nietos y los padres en la sociedad actual. Recuperado de: http://www.eumed.net/rev/ cccss/10/almm.htm

Mukherjee, A. y Parasuraman, S. (2012). Transmission of inter-generational spousal violence against women in India. Paper to be presented at Population Association of America 2012 Annual Meeting, for session N. ${ }^{\circ}$ 110: Domestic Violence: Causes and Consequences.

OMS. (2005). Estudio multipaís de la OMS sobre salud de la mujer y violencia doméstica contra la mujer, primeros resultados sobre prevalencia, eventos relativos a la salud y respuestas de las mujeres a dicha violencia: resumen del informe. Recuperado de: http://whqlibdoc. who.int/publications/2005/924359351X_spa.pdf

Pardo, A. (2008). Legado transgeneracional y resiliencia. Recuperado de: http://www.avntf-evntf.com/imagenes/biblioteca/Pardo,\%20A.\%20Tbjo.\%203\%C2\%BA\%20online\%2008.pdf

Ramírez, B., Ospina, D. (1998). Efectos psicológicos de la violencia por conflicto armado en la zona del Urabá antioqueño: Una sistematización del programa de intervención psicosocial. Rev. Ciudad Don Bosco, 1(7), 48-54.

Salazar, D. (2010). Mediación y violencia de pareja. (Tesis para optar el grado de Magíster en Psicología Jurídica y Forense). Universidad de la Frontera, Temuco, México.

Sánchez, M., Manzo, M. (2014). La violencia conyugal y su transmisión transgeneracional. Uaricha, 11(24), 68-77.

Schützenberger, A. (2007). Ay, mis ancestros. Recuperado de: http://editorialtaurus.com/ uploads/ficheros/libro/primeras-paginas/200801/primeras-paginas-ay-mis-ancestros. pdf

Segoviano, M. (2008). Transmisión psíquica escuela francesa. Psicoanálisis e intersubjetividad, 3. Recuperado de: http://www.intersubjetividad.com.ar/website/articulo.asp?i$\mathrm{d}=202$ yidd $=3$

Stith, S., Rosen, K., Middleton, K., Busch, A., Lundeberg, K., Carlton, R. (2004). The Intergenerational Transmission of Spouse Abuse: A Meta-Analysis. Journal of Marriage and Family, 62(3), 640-654. 
Straus, M., Gelles, R., Steinmetz, S. (1980). Behind closed doors: violence in the American family. New York. Anchoor Doubleday.

Tapia, M., Peréz Vélez, N. (2011). La transmisión transgeneracional del psiquismo. Rev Psi Uaricha, 8(16), 45-52.

Tisseron, S., Torok, M., Rand, M. (1997). El psiquismo ante la prueba de las generaciones. Argentina: Amorrortu.

Tuesca, R., Borda, M. (2003). Violencia física marital en Barranquilla (Colombia): prevalencia y factores de riesgo. Gac Sanit, 17(4), 302-308.

Urzagasti, O. A. (2006). Estructura de la familia de origen y nuclear en varones que ejercen violencia física en el ámbito conyugal. Ajayu, 4(2), 268-289. 